\title{
Thermal Radiation and Heat Transfer Effects on MHD Micro polar Fluid Flow Past a Vertical Plate with Chemical Reaction
}

\author{
K.BhagyaLakshmi ${ }^{1}$, P.V.Satya Narayana ${ }^{2}$ and N.V.R.V.Prasad ${ }^{3}$ \\ ${ }^{I}$ (Department of Mathematics, CMR Technical Campus, Medchel, Hyderabad,Telangana,India) \\ ${ }^{2}$ (Department of Mathematics, SAS, VIT University, Vellore 632 014, T.N, India) \\ ${ }^{3}$ (Department of Mathematics, S.V.G.S. Junior College, Nellore,A.P, India)
}

\begin{abstract}
We examine the instantaneous effects of chemical reaction and radiation absorption on unsteady MHD free convective heat and mass transfer flow for a micro polar fluid bounded by a semi infinite vertical plate in the presence of heat generation and thermal radiation. The plate is assumed to move with a constant velocity in the direction of fluid flow. A uniform magnetic field acts perpendicular to the porous surface in which absorbs micro polar fluid with a suction velocity varying with time. The dimensionless governing equations of the flow, heat and mass transfer are solved analytically using regular perturbation method. The effects of various pertinent parameters on flow, heat and mass transfer properties are discussed analytically and explained graphically. Also the velocity profiles of micro polar fluid is compared with the corresponding flow problem for a Newtonian fluid and found that the polar fluid velocity is decreasing.

Keywords: Chemical reaction, heat transfer, micro polar fluid, $M H D$, thermal radiation
\end{abstract}

\section{Introduction}

The theory of micropolar fluids was first introduced and formulated by Eringen [1]. This theory displays the effects of local rotary inertia and couple stress. The theory is expected to a mathematical model for the non-Newtonian fluid behavior observed in certain fluid such as exotic lubricants, colloidal fluids, liquid crystals etc., which is more realistic and important from a technological point of view. The theory of thermo micropolar fluids was developed by Eringen [2] by extending his theory of micropolar fluid. Sharma and Gupta [3] studied the effects of medium permeability on thermal convection in micropolar fluids. Prathap Kumar et al. [4] have studied the problem of fully developed free convective flow of micropolar and viscous fluids in a vertical channel. Muthu et.al. [5] studied peristaltic motion of micropolar fluid in circular cylindrical tubes. Srinivasacharya et.al. [6] analyzed the unsteady stokes flow of micropolar fluid between two parallel porous plates. Muthuraj and Srinivas [7] investigated, fully developed MHD flow of a micropolar and viscous fluids in a vertical porous space using HAM. Kim [8] investigated the effects of heat and mass transfer in the MHD micropolar fluid flow past a vertical moving plate.

The role of thermal radiation on the flow and heat transfer process is major importance in the design of many advanced energy conversion systems operating at higher temperatures. Thermal radiation within the system is the result of emission by hot walls and the working fluid. Effects of chemical reaction and thermal radiation on heat and mass transfer flow of MHD micropolar fluid in rotation frame of reference is investigated by Das [9]. A complete analytic solution to heat transfer of a micropolar fluid through a porous medium was analyzed by Rashidi et al. [10].MHD flow of a micropolar fluid towards a vertical permeable plate with prescribed surface heat flux is investigated by Nor Azizah et al. [11]. Ezzat et al. [12] steadied the combined heat and mass transfer for unsteady MHD flow of perfect conducting micropolar fluid with thermal relaxation. Peristaltic motion of a magneto hydrodynamic micro polar fluid in tube is analyzed by Yongqi Wang et al. [13]. Patil , Kulkarni [14] studied the effects of chemical reaction on free convective flow of a polar fluid through a porous medium in the presence of internal heat generation. Kesavaiah et.al, [15] investigated, effects of the chemical reaction and radiation absorption on an unsteady MHD convective heat and mass transfer flow past a semi-infinite vertical permeable moving plate. Effects of chemical reaction on unsteady MHD heat and mass transfer flow past a semi infinite vertical porous moving plate in the presence of viscous dispation is analyzed by Bhagya Lakshmi[16]. Many researchers [17-20] have studied the problem of non-Newtonian fluid flows analytically and numerically over various flow geometries.

In this paper we examine the instantaneous effects of chemical reaction and radiation absorption on unsteady MHD free convective heat and mass transfer flow for a micropolar fluid bounded by a semi infinite vertical plate in the presence of heat generation and thermal radiation. The plate is assumed to move with a constant velocity in the direction of fluid flow. A uniform magnetic field acts perpendicular to the porous surface in which absorbs micropolar fluid with a suction velocity varying with time. The dimensionless

International Conference on Recent Innovations in Civil \& Mechanical Engineering $\quad 7 \mid$ Page [i-CAM2K16] DOI: 10.9790/1684-16053030714 
governing equations of the flow, heat and mass transfer are solved analytically using regular perturbation method. The effects of various pertinent parameters on flow, heat and mass transfer properties are discussed analytically and explained graphically. Also the velocity profiles of micropolar fluid is compared with the corresponding flow problem for a Newtonian fluid and found that the polar fluid velocity is decreasing.

\section{Formulation}

An unsteady two-dimensional hydromagnetic laminar free convection with mass transfer flow of an incompressible, electrically conducting micropolar fluid past a semi-infinite vertical permeable moving plate embedded in a saturated porous medium in the presence of thermal radiation and chemical reaction is considered. The $x^{*}$-axis measured along the porous plate, in the upward direction and a magnetic field of uniform strength $B_{0}$ is applied in the $y^{*}$-direction which is normal to the flow direction. Since, the motion is two dimensional and length of the plate is very large so all the physical variables are independent of $x^{*}$ and they are functions of normal distance $y^{*}$ and $t^{*}$ only. It is assumed that there is no applied voltage which implies the absence of an electrical field. The transversely applied magnetic field and magnetic Reynolds number are assumed to be very small so that the induced magnetic field is negligible. It is assumed that the hole size of the porous plate is significantly larger than a characteristic microscopic length scale of the porous medium. The suction velocity is assumed to be $v^{*}=-V_{0}\left(1+\varepsilon A e^{\delta^{*} t^{*}}\right)$ where $A$ is a real positive constants and $\varepsilon$ and $\varepsilon A$ are small values less than unity, and $V_{0}$ is scale of suction velocity which is non-zero positive constant. The negative sign indicates that the suction is towards the plate. The fluid properties are assumed to be constant except that the influence of density variation with temperature and concentration has been considered in the body-force term. The concentration of diffusing species is very small in comparison to other chemical species, the concentration of species far from the wall $C_{\infty}^{*}$, is infinitesimally small and hence the Soret and Dufour effects are neglected. The chemical reactions are taking place in the flow and all thermo physical properties are assumed to be constant.

Under these conditions, the governing equations can be written in a Cartesian frame of reference as:

$$
\begin{aligned}
& \frac{\partial v^{*}}{\partial y^{*}}=0 \\
& \frac{\partial u^{*}}{\partial t^{*}}+v^{*} \frac{\partial u^{*}}{\partial y^{*}}=-\frac{1}{\rho} \frac{\partial P^{*}}{\partial x^{*}}+\left(v+v_{r}\right) \frac{\partial^{2} u^{*}}{\partial y^{* 2}}+g \beta_{f}\left(T^{*}-T_{\infty}^{*}\right)+g \beta_{c}\left(C^{*}-C_{\infty}^{*}\right) \\
& -v \frac{u^{\prime}}{K_{0}}-\frac{\sigma}{\rho} B^{2} u^{*}+2 v_{r} \frac{\partial \omega^{*}}{\partial y^{*}} \\
& \rho j^{*}\left(\frac{\partial \omega^{*}}{\partial t^{*}}+v^{*} \frac{\partial \omega^{*}}{\partial y^{*}}\right)=\gamma \frac{\partial^{2} \omega^{*}}{\partial y^{* 2}} \\
& \frac{\partial T^{*}}{\partial t^{*}}+v^{*} \frac{\partial T^{*}}{\partial y^{*}}=\frac{K}{\rho c_{p}} \frac{\partial^{2} T^{*}}{\partial y^{* 2}}+\frac{Q_{0}}{\rho c_{p}}\left(T^{*}-T_{\infty}^{*}\right)+Q_{l}^{*}\left(C^{*}-C_{\infty}^{*}\right)-\frac{1}{\rho c_{p}} \frac{\partial q_{r}^{*}}{\partial y^{*}} \\
& \frac{\partial c^{*}}{\partial t^{*}}+v^{*} \frac{\partial c^{*}}{\partial y^{*}}=D^{*} \frac{\partial^{2} c^{*}}{\partial y^{* 2}}-R^{*}\left(C^{*}-C_{\infty}^{*}\right)
\end{aligned}
$$
fields are

The appropriate boundary conditions for the velocity, angular velocity, temperature and concentration

$$
\begin{aligned}
& u^{*}=u_{p}^{*}, T^{*}=T_{w}^{*}+\varepsilon\left(T_{w}^{*}-T_{\infty}^{*}\right) e^{\delta^{*} t^{*}}, \\
& \omega^{*}=-n \frac{\partial u^{*}}{\partial y^{*}}, C^{*}=C_{w}^{*}+\varepsilon\left(C_{w}^{*}-C_{\infty}^{*}\right) e^{\delta^{*} t^{*}} \text { at } \mathrm{y} \rightarrow 0 \\
& u^{*} \rightarrow U_{\infty}^{*}=U_{0}\left(1+\varepsilon e^{\delta^{* *}}\right), T^{*} \rightarrow T_{\infty}^{*}, C^{*} \rightarrow C_{\infty}^{*}, \omega^{*} \rightarrow 0 \text { at } \mathrm{y} \rightarrow \infty
\end{aligned}
$$

in which $t^{*}$ is the time, $\delta^{*}$ is a scalar constant, $U_{0}$ is a scale of free stream velocity.

Outside the boundary layer, equation (2) gives 
$-\frac{1}{\rho} \frac{\partial P^{*}}{\partial x^{*}}=\frac{d U_{\infty}^{*}}{d t^{*}}+\frac{v}{K^{*}} U_{\infty}^{*}+\frac{\sigma}{\rho} B_{0}^{2} U_{\infty}^{*}$

The radiative heat flux term by using the Rosseland approximations given by

$q_{r}^{*}=\frac{4 \sigma^{*}}{3 K_{1}^{*}} \frac{\partial T^{* 4}}{\partial y^{*}}$

Where $\quad T^{* 4}=4 T_{\infty}^{* 3}-3 T_{\infty}^{* 4}$

We introduce the dimensionless variable as follows:

$\mathrm{u}^{*}=\mathrm{U}_{0} \mathrm{u}, v^{*}=V_{0} v, u_{p}^{*}=U_{0} U_{p}, y^{*}=\frac{v y}{V_{0}}, U_{\infty}^{*}=U_{0} U_{\infty}, \omega^{*}=\frac{\omega U_{0} V_{0}}{V_{0}}$,

$j^{*}=\frac{j v^{2}}{V_{0}^{2}}, t^{*}=\frac{t v}{V_{0}^{2}}, T^{*}=T_{\infty}^{*}+\theta\left(T_{w}^{*}-T_{\infty}^{*}\right), c^{*}=c_{\infty}^{*}+c\left(c_{w}^{*}-c_{\infty}^{*}\right), \delta^{*}=\frac{\delta v_{0}^{*}}{v}, K^{*}=\frac{K v_{0}^{2}}{V_{0}^{2}}$,

$s_{c}=\frac{v}{D^{*}}, H=\frac{v Q_{0}}{\rho v c_{p}}, \quad \zeta=\frac{R^{*} v}{V_{0}^{2}} M=\frac{v \sigma B_{0}^{2}}{\rho V_{0}^{2}}, G r=\frac{v g B_{f}}{U_{0} V_{0}^{2}}\left(T_{w}^{*}-T_{\infty}^{*}\right), G c=\frac{v g B_{c}}{U_{0} V_{0}^{2}}\left(c_{w}^{*}-c_{\infty}^{*}\right)$,

$\operatorname{Pr}=\frac{v \rho c_{p}}{K}=\frac{v}{\alpha}, R=\frac{4 T_{\infty}^{* 2} \sigma^{*}}{K K_{1}^{*}}$

$\alpha=\frac{K}{\rho c_{p}}$

Furthermore, the spin-gradient viscosity $\gamma$ which, gives same relationship between the coefficients of viscosity and micro inertia is defined as

$\gamma=\left(\mu+\frac{\Gamma}{2}\right) j^{*}=\mu j^{*}\left(1+\frac{\beta}{2}\right)$

where, $\beta=\frac{\Gamma}{\mu}$.

In view of equations (9) - (13), the governing equations (2) - (5) reduce to the following dimensionless form:

$\frac{\partial u}{\partial t}-\left(1+\varepsilon A e^{\delta t}\right) \frac{\partial u}{\partial y}=\frac{d u_{\infty}}{d t}+(1+\beta) \frac{\partial^{2} u}{\partial y^{2}}+G r \theta+G c C++\frac{\left(U_{\infty}-u\right)}{K}+M\left(U_{\infty}-u\right)+2 \beta \frac{\partial \omega}{\partial y}$

$\frac{\partial \omega}{\partial t}-\left(1+\varepsilon A e^{\delta t}\right) \frac{\partial \omega}{\partial y}=\frac{\partial^{2} \omega}{\partial y^{2}}$

$\frac{\partial \theta}{\partial t}-\left(1+\varepsilon A e^{\delta t}\right) \frac{\partial \theta}{\partial y}=\frac{1}{\operatorname{Pr}}\left(1+\frac{4 R}{3}\right) \frac{\partial^{2} \theta}{\partial y^{2}}+H \theta+Q l c$

$\frac{\partial c}{\partial t}-\left(1+\varepsilon A e^{\delta t}\right) \frac{\partial c}{\partial y}=\frac{1}{S c} \frac{\partial^{2} c}{\partial y^{2}}-\zeta c$

Where $\eta=2 /(2+\beta)=\mu j^{*} / \gamma$

The boundary conditions (6) and (8) are then given by the following dimensionless form:

$$
\begin{aligned}
& u=U_{P}, \theta=1+\varepsilon e^{\delta t}, \omega=-n \frac{\partial u}{\partial y}, c=1+\varepsilon e^{\delta t} \quad \text { at } \mathrm{y} \rightarrow 0 \\
& u=U_{\infty} \rightarrow\left(1+\varepsilon e^{\delta t}\right), \theta \rightarrow 0, \omega \rightarrow 0, c \rightarrow 0 \quad \text { at } \mathrm{y} \rightarrow \infty
\end{aligned}
$$




\section{Solution of The Problem}

In order to reduce the above system of partial differential equations in dimensionless form, we may represent the linear and angular velocities, temperature and concentration as:

$$
\begin{aligned}
& u=u_{0}(y)+\varepsilon e^{\delta t} u_{1}(y)+o\left(\varepsilon^{2}\right) \\
& \omega=\omega_{0}(y)+\varepsilon e^{\delta t} \omega_{1}(y)+o\left(\varepsilon^{2}\right) \\
& \theta=\theta_{0}(y)+\varepsilon e^{\delta t} \theta_{1}(y)+o\left(\varepsilon^{2}\right) \\
& c=c_{0}(y)+\varepsilon e^{\delta t} c_{1}(y)+o\left(\varepsilon^{2}\right)
\end{aligned}
$$

By substituting the above equations (21) - (24) into equations (14) - (17), equating the harmonic and nonharmonic terms and neglecting the higher-order terms of $\mathrm{O}\left(\varepsilon^{2}\right)$, we obtain the following equations. Zeroth- order:

$$
\begin{aligned}
& (1+\beta) u_{0}{ }^{\prime \prime}+u_{0}^{\prime}-N u_{0}=-N-G r \theta_{0}-G c c_{0}-2 \beta \omega_{0}^{\prime} \\
& \omega_{0}^{\prime \prime}+\eta \omega_{0}=0 \\
& (3+4 R) \theta_{0}^{\prime \prime}+3 \operatorname{Pr}\left(\theta_{0}^{\prime}+H \theta_{0}\right)=-3 \operatorname{Pr} Q_{l} c_{0} \\
& c_{0}^{\prime \prime}+S c c_{0}^{\prime}-S c c_{0}=0
\end{aligned}
$$

First-order:

$$
\begin{aligned}
& (1+\beta) u_{1}^{\prime \prime}+u_{1}^{\prime}-(N+\delta) u_{1}=-u_{0}-G r \theta_{1}-G c c_{1}-2 \beta \omega_{1}^{\prime}-A u_{0}^{\prime} \\
& \omega_{1}^{\prime \prime}+\eta \omega_{1}^{\prime}-\delta \eta \omega_{1}=A \eta \omega_{0}^{\prime} \\
& (3+4 R) \theta_{1}^{\prime \prime}+3 \operatorname{Pr} \theta_{1}^{\prime}-3 P_{r}(\delta-H) \theta_{1}=-3 A \operatorname{Pr} \theta_{0}^{\prime}-3 \operatorname{Pr} Q_{l} c_{1} \\
& c_{1}^{\prime \prime}+S c c_{1}-S c c_{1}=-A S c c_{0}^{\prime}
\end{aligned}
$$

Where $\mathrm{N}=(\mathrm{M}+1 / \mathrm{K})$ the primes denote differentiation with respect to $y$.

The corresponding boundary conditions can be written as

$$
\begin{gathered}
u_{0}=U_{P}, u_{1}=0, \omega_{0}=-n u_{0}^{\prime}, \omega_{1}=-n u_{1}^{\prime}, \theta_{0}=1, \theta_{1}=1, c_{0}=1, c_{1}=1 \text { at } \mathrm{y} \rightarrow 0 \\
u_{0}=1, u_{1}=1, \omega_{0}=0, \omega_{1}=0, \theta_{0}=0, \theta_{1}=0, c_{0}=0, c_{1}=0 \quad \text { at } \mathrm{y} \rightarrow \infty
\end{gathered}
$$

The solutions of equations (25)-(32) with satisfying boundary conditions (33) and (34) are given by

$$
\begin{aligned}
& u_{0}(y)=1+a_{1} e^{-R_{1} y}+a_{2} e^{-b_{3} y}+a_{6} e^{-b_{1} y}+a_{5} e^{-\eta y} \\
& c(y, t)=c_{0}(\mathrm{y})+\varepsilon e^{\delta t} c_{1}(\mathrm{y}) \\
& \omega_{0}(y)=c_{11} e^{-\eta y} \\
& \omega_{1}(y)=c_{13} e^{-h_{6} y}-\frac{A \eta c_{11}}{\delta} e^{-\eta y} \\
& \theta_{0}(y)=\left(1-z_{3}\right) e^{-b_{3} y}+z_{3} e^{-b_{1} y} \\
& \theta_{1}(y)=c_{9} e^{-h_{4} y}+z_{4} e^{-b_{3} y}+z_{6} e^{-b_{2} y}+z_{8} e^{-b_{1} y} \\
& c_{0}(y)=e^{-h_{1} y} \\
& c_{1}(y)=\left(1-z_{3}\right) e^{-b_{2} y}+z_{2} e^{-b_{1} y}
\end{aligned}
$$

The Skin friction, Couple stress coefficient ,Nusselt number and Sherwood number are given by

$$
c_{f}=\frac{2 \tau_{w}^{*}}{\rho V_{0} U_{0}}=2[1+(1-n) \beta] u^{\prime}(0) \quad c_{m}=\frac{M_{w}}{\mu j U_{0}}=\left(1+\frac{\beta}{2}\right) \omega^{\prime}(0)
$$

$$
N u_{x} R e_{x}^{-1}=-\left.\left(1+\frac{4 R}{3}\right) \frac{\partial \theta}{\partial y}\right|_{y=0} \quad S h_{x} \operatorname{Re}_{x}^{-1}=-\left.\left(\frac{\partial c}{\partial y}\right)\right|_{y=0}
$$

Where $\operatorname{Re}_{x}=\frac{v_{0} x}{v}$ 


\section{Results And Discussions}

For different values of the magnetic field parameter $\mathrm{M}$, the translational velocity and microrotation profiles are plotted in Fig. 1 and 2. It is obvious that the effect of increasing values of the parameter M results in a decreasing velocity distribution across the boundary layer. The effect of magnetic field is more prominent at the point of peak value i.e. the peak value drastically decreases with increases in the value of magnetic field, because the presence of magnetic field in an electrically conducting fluid introduce a force called the Lorentz force, which acts against the flow if the magnetic field is applied in the normal direction, as in the present problem. This type of resisting force slows down the fluid velocity as shown in this figure. The results also show that the magnitude of the microrotation on the porous plate increases as $\mathrm{M}$ increases.

Figure 3 and 4 illustrates the variation of velocity and microrotation distribution across the boundary layer for several values of plate moving velocity $U_{p}$ in the direction of the fluid flow, respectively. Although we have different initial plate moving velocities, the velocity decrease to the constant values for given material parameters. The results also show that the magnitude of microrotation on porous plate increases as $U_{p}$ increases.

For various values of $Q_{l}$, the profiles of translational velocity and the microrotation across the boundary layer are shown in Fig.5 and 6. It is clear from the graph that the translational velocity increases with an increase of $Q_{l}$, which is due to the fact that when heat is absorbed the buoyancy force which accelerated the flow rate. The results also show that the magnitude of microrotation on porous plate decreases as $Q_{l}$ increases.

The effects of heat generation $H$ on the translational velocity and microrotation profiles across the boundary layer are presented in Fig .7 and 8. It is shown that the translational velocity across the boundary layer increases with an increasing of $H$. The results also show that the magnitude of microrotation decreases with an increasing of $H$.

Fig.9 and 10 represents the temperature profiles for various values of thermal radiation parameter $\mathrm{R}$ and prandtl number Pr in the boundary layer. This figure indicate that the effect of thermal radiation is to enhance heat transfer because of the fact that thermal boundary layer thickness increases with increase in the thermal radiation. Thus it is point out that the radiation should be minimized to have the cooling process at a faster rate. But the temperature decreases with increasing the values of Prandtl number Pr in the boundary layer. From this plot it is evident that temperature in the boundary layer falls very quickly for large value of the Prandtl number, because of the fact that thickness of the boundary layer decreases with increase in the value of the Prandtl number.

Typical variations of the temperature profiles along the spanwise coordinate $y$ are shown in Fig. 11 for varies values of $Q_{l}$.The results show that as an increasing $Q_{l}$, the temperature profiles decreases .The temperature profiles with respect to span wise coordinate $y$ for various values of $\zeta$ is shown in Fig.12.The results show that as an increasing of the chemical reaction parameter $\zeta$, the thermal boundary layer increases.

Figure 13 and 14 presents typical profiles of the velocity and angular velocity for various values of Schmidt number $S c$. It is noted from figure that an increase in the value of $S c$, leads to a decrease in the velocity of fluid. Further it is observed that the velocity of the polar fluid is found to decrease in comparison with Newtonian fluid ( $\alpha=0$ and $\beta=0$ ). This is due to the fact that as the Schmidt number increases the concentration decreases which leads to decrease in concentration buoyancy effect, hence a decrease in fluid velocity. The opposite behavior is found in the case of angular velocity. The negative values of the angular velocity indicate that the micro rotation of sub structures in the polar fluid is clock-wise.

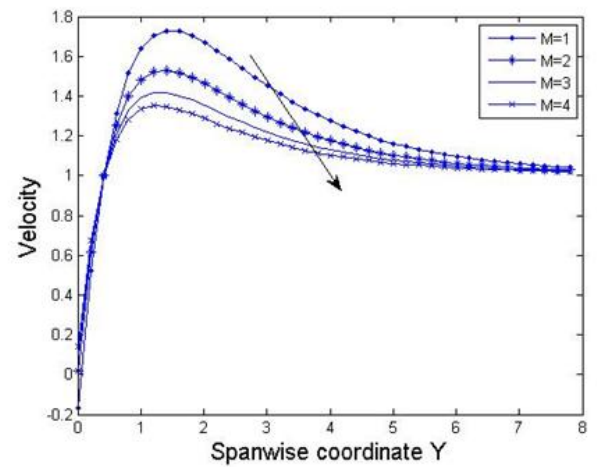

Fig.1. Velocity profiles for various values of $\mathrm{M}$

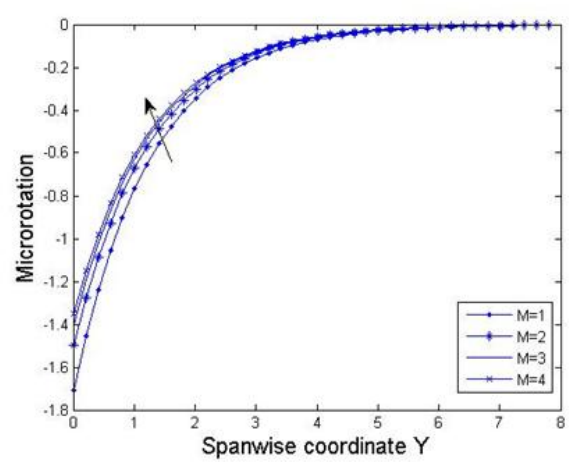

Fig.2. Micro-rotation profiles for various values of $\mathrm{M}$ 


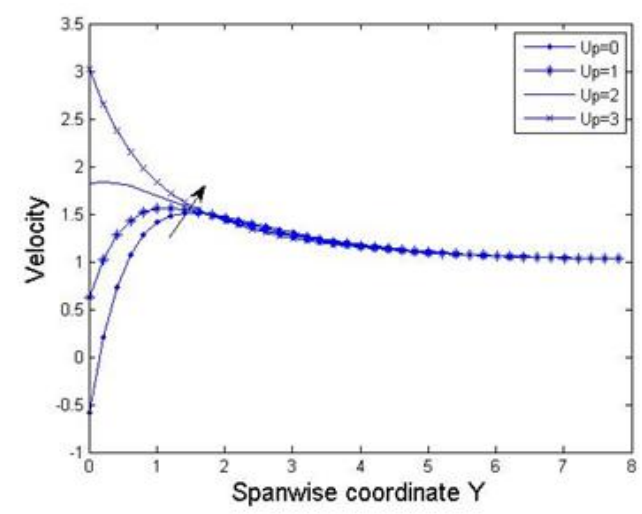

Fig.3. Velocity profiles for various values of $U p$

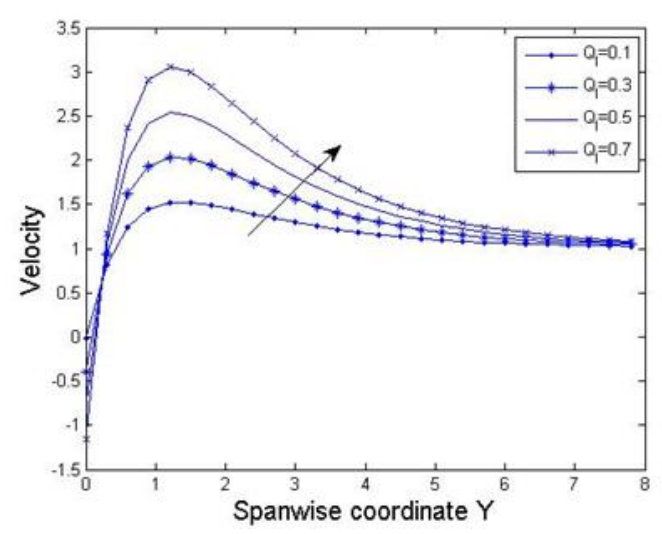

Fig.5. Velocity profiles for various values of $\mathrm{Q}_{1}$

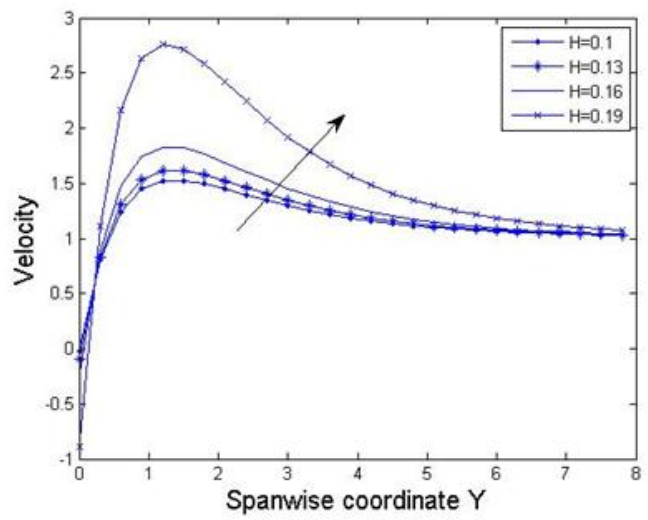

Fig.7. Velocity profiles for various values of $\mathrm{H}$

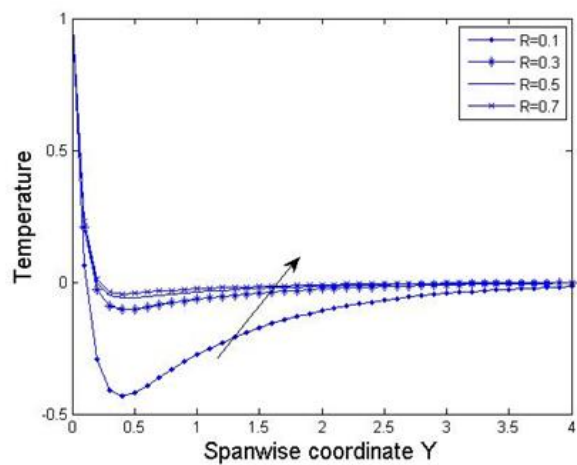

Fig.9. Temperature profiles for various values of $\mathrm{R}$

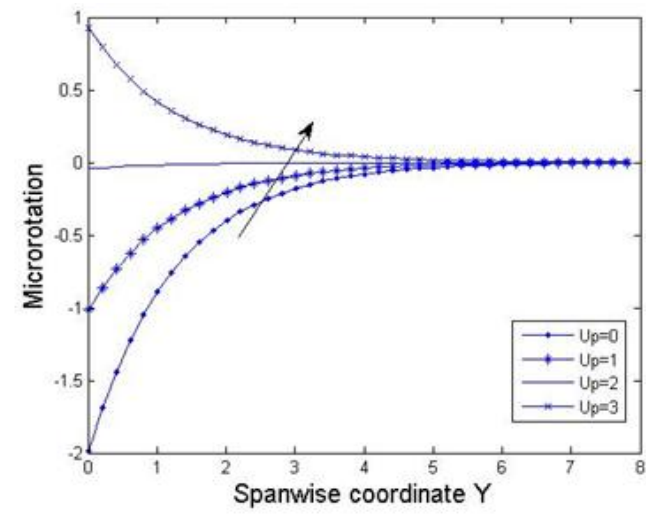

Fig.4. Micro-rotation profiles for various values of Up

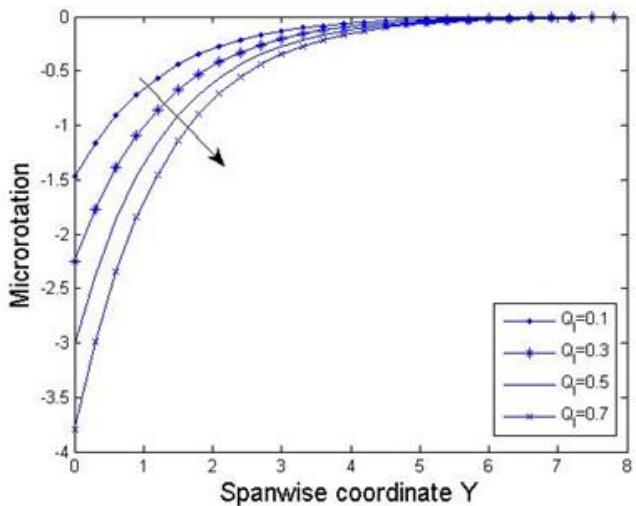

Fig.6. Micro-rotation profiles for various values of $\mathrm{Q}_{1}$

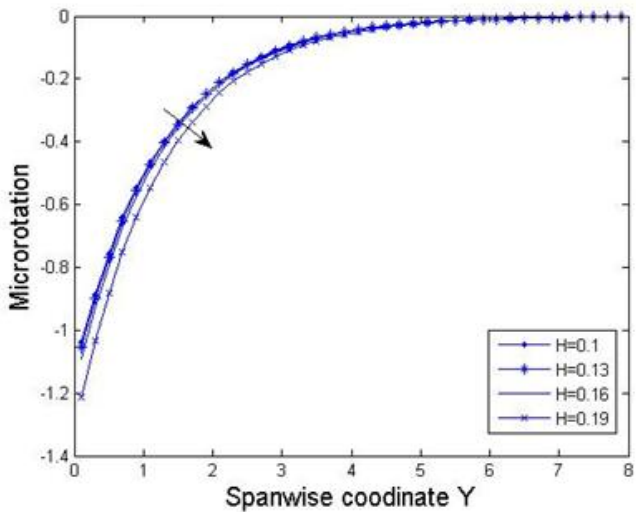

Fig.8. Micro-rotation profiles for various values of $\mathrm{H}$

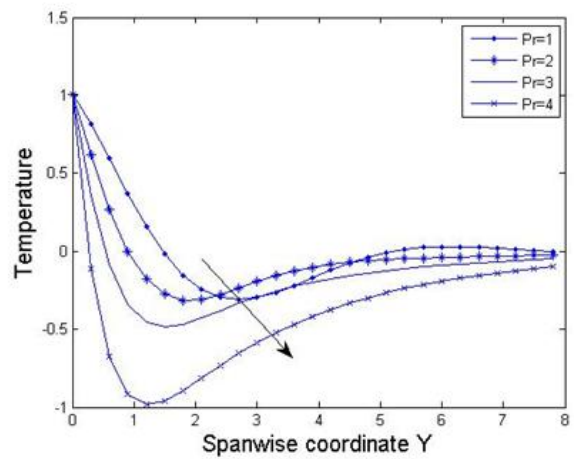

Fig.10. Temperature profiles for various values of $\operatorname{Pr}$ 


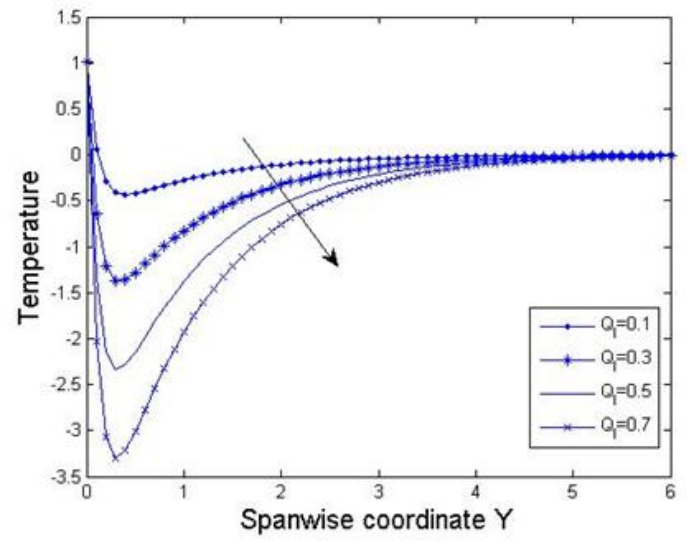

Fig.11. Temperature profiles for various values of $\mathrm{Q}_{1}$,

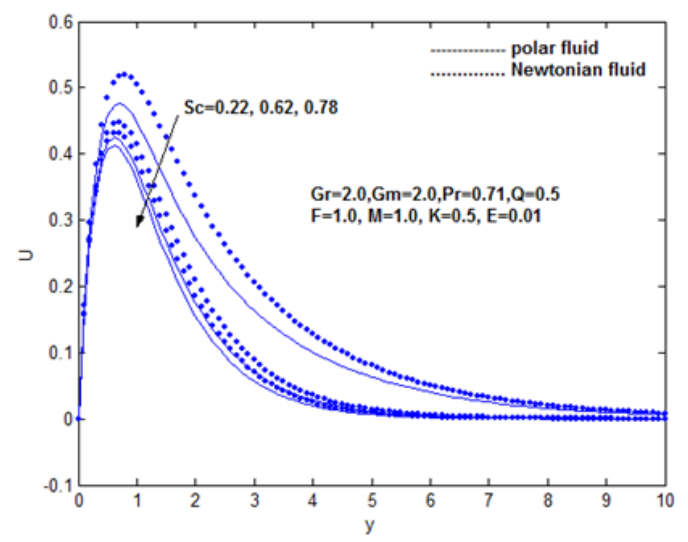

Fig. 14. Effects of Schimidt number Sc on velocity profiles.

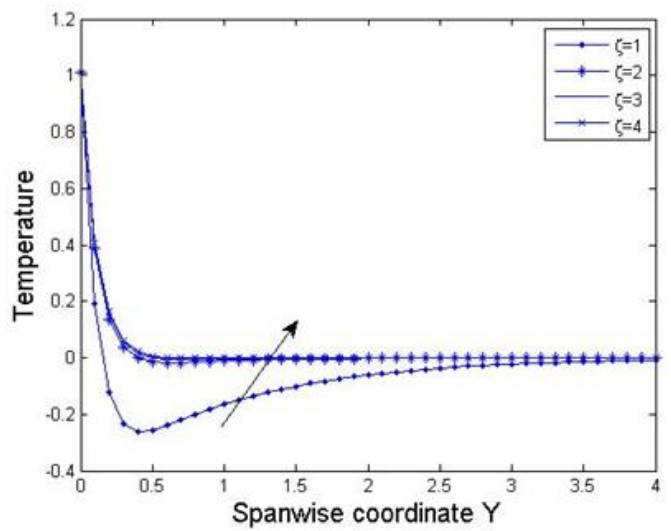

Fig.12. Temperature profiles for various values of $\zeta$

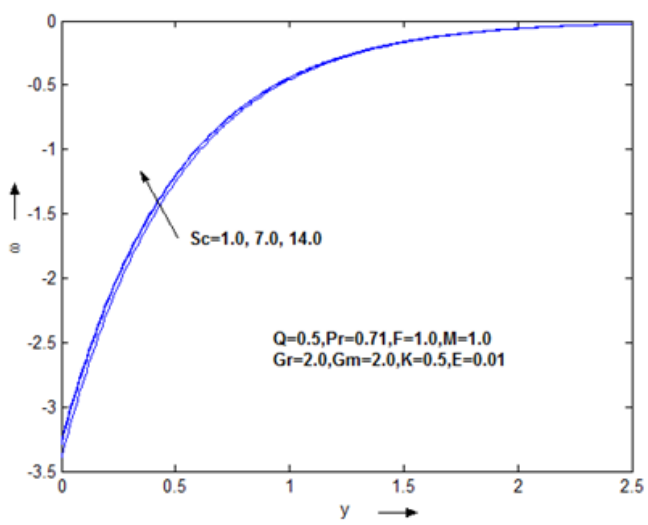

Fig. 13. Effects of Schimidt number Sc on angular velocity profiles

\section{References}

[1]. Eringen A.C. Theory of micropolar fluids, J.Math.Mech.Vol 16 (1966) pp.1-18.

[2]. Eringen A.C., J.Math.Anal.Appl.Vol 38(1972) pp 480-496.

[3]. R.C.Sharma, U.Gupta, Thermal Convection in micropolar fluids in porous medium, Int.J.Eng.Sci.33 (1995) 18871892.

[4]. J. Pratap kumar, J.C.Umavathi, J.Chamkha Ali, I.Pop, Fully developed free convective flow of micropolar and viscous fluids in a vertical channel, Applied Mathematical Modeling, 34 (2010) 1175-1186.

[5]. P.Muthu, B.V.Rathish Kumar, Peeyush Chandra, Peristalic motion of micropolar fluid in circular cylindrical tubes: Effect of wall properties, Applied Mathematical Modelling, 32(2008) 2019-2033.

[6]. D.Srinivacharya, J.V.Ramana murthy, D.Venugopalam, Unsteady Stokes flow of micropolar fluid between two parallel porous plates, Int.J.Engg,Sci.39(2001) 1557-1563.

[7]. R. Muthuraj, S.Srinivas, Fully developed MHD flow of a micropolar and viscous fluids in a vertical porous space using HAM, Int.J.of Appl. Math and Mech. 6(2010) 55-78.

[8]. Y.J.Kim, Heat and mass transfer in MHD micropolar flow over a vertical moving plate in a porous medium, J.Trans Porous Media, 56(2004) 17-37.

[9]. K. Das, Effects of chemical reaction and thermal reaction on heat and mass transfer flow of MHD micropolar fluid in a rotating frame of reference, International Journal of heat and mass mass transfer, 54(2011) 3505-3513.

[10]. M.M. Rashid, S.A. Mohimanian Pour, S.Abbasbandy, Analytic approximate solutions for heat transfer of a micropolar fluid through a porous medium with radiation, Communications in Non-linear science and numeric al simulation, 16(2011) 1874-1889.

[11]. Nor Azizah, Anular Ishak, MHD flow of a micropolar fluid towards a vertical permeable plate with prescribed surface heat flux, Chemical Engineering Research and Design, 89(2011) 2291-2297.

[12]. M.Ezzat, A.A. Ei-Bary, S. Ezzat, Combined heat and mass transfer for unsteady MHD flow of perfect conducting micropolar fluid with thermal relaxation, Energy conversion and management, 52(2011) 934-945.

[13]. Yongqi Wang, Nasir Ali, Tasawaar Hayat, Martin Oberlack, Peristalic motion of a magnetohydrodynamic micropolar fluid in a tube, Applied Mathematical modeling, 35(2011) 3737-3750.

[14]. P.M. Patil, P.S. Kulkarni, Effects of chemical reaction on free convective flow of a polar fluid through a porous medium in the presence of internal heat generation, International journal of thermal sciences, 47(2008) 1043-1054.

International Conference on Recent Innovations in Civil \& Mechanical Engineering

13 | Page 
[15]. D.Ch Kesavaiah, P.V. Satya Narayana and S.Venkataramana, Effects of the chemical reaction and radiation absorption on an unsteady MHD convective heat and mass transfer flow past a semi-infinite vertical permeable moving plate embedded in a porous medium with heat source and suction" Int. J. of Appl. Math and Mech. 7 (1)(2011) 52-69.

[16]. K. Bhagya Lakshmi, G.S.S. Raju, N.V.R.V. Prasad, Effects of chemical reaction on unsteady MHD Heat \& Mass Transfer flow past a semi-infinite vertical porous moving plate in the presence of viscous dispation, International Journal of Management, IT and Engineering:3(5)(2013) 111-130.

[17]. D. Harish Babu, P.V. Satya Narayana, Joule heating effects on MHD mixed convection of a Jeffrey fluid over a stretching sheet with power law heat flux: a numerical study. Journal of Magnetism and Magnetic Materials. 412 (2016) 185-193.

[18]. P.V. Satya Narayana, D. Harish Babu . Numerical MHD heat and mass transfer Jeffrey fluid over a stretching sheet with chemical reaction and radiation parameter. Journal of the Taiwan Institute of Chemical Engineers,59 (2015)1825 .

[19]. P. V. Satya Narayana, B. Venkateswarlu and S. Venkataramana, Effects of Hall current and radiation absorption on MHD micropolar fluid in a rotating system. Ain Shams Engineering Journal, 4(4) (2013) 843-854.

[20]. PV Satya Narayana and B Venkateswarlu, Heat and mass transfer on MHD nanofluid flow past a vertical porous plate in a rotating system. Frontiers in Heat and Mass Transfer, (2016), 7:8 